\title{
CRECIMIENTO Y CITOCINESIS DE STAURASTRUM PLANCTONICUM TEILING VAR. BULLOSUM TEILING (CHLOROPHYCEAE, DESMIDIACEAE) EN UN EMBALSE EUTROFICO
}

\author{
M. Araúzo Sánchez, , M. Alvarez Covelas, A. Rubio Olmo \\ Centro de Investigaciones del Agua (C.S.I.C.). Madrid.
}

Palabras clave: Cytokinesis, population, growth. loss, rate.

\author{
ABSTRACT \\ GROWTH AND CYTOKINESIS O F STAUR ISTRLM PLANCTONICUM TEILING VAR. \\ $B I ' L L O S U M$ TEILING (CHLOROPHYCEAE DESMIDIACEAE) \\ IN AN EUTROPHIC RESERVOIR
}

\begin{abstract}
Cell division (only cytokinetic phase) of Siaurastmm planc tonk'um var. hullosum (D) smidiaceas) is studied in tlie epilimnion of an eutrophic reservoir (El Vellón. Madrid) for two days close to each other but with contrasting weather conditions. Populationbased growth rates. which reflect both advection and sedimentation processes experienced by cells in the water column. are compared with cytokinefic time-based growth rates, which do not depend on such proceses. On tlie first day population-based growth nites was $0.04 \mathrm{day}^{-}$whilst it was 4.35 day ori the second. Cytokinetic-based growth rates were 0.12 on both dates. Loss rates were computed from the difference between cy tokinetic-based and population-based growth rntes. accounting for 0.08 day $^{-1}$ and 0.47 $\mathrm{das}^{-1}$ respectively. Advection processes uere more important controlling losses on the first day than on tlie second when sedimentation played a key role on losing. It is suggested that weather conditions might influence cytokinetic time ori tlie basis of different spans ori two dates under study. Some methodological conclusions are produced concerning sampling designs on phytoplankton growth studies in the field
\end{abstract}

\section{INIRODUCCION}

A diferencia de los cultivos. donde puede ser calculada directamente a partir de las variaciones en la densidad de población. el cálculo de la tasa de crecimiento en poblaciones naturales resulta complicado por la existencia de procesos de pérdida. En los últimos años algunos autores han realizado estudios sobre la tasa de crecimiento en dinoflagelados a partir del tiempo de división (bien sea el de mitosis o el de citocinesis), cálculo posible unicamente cuando se pueden distinguir al microscopio fases definidas en dicho periodo. McDuff \& Chisholm (1982) hacen una revisión sobre los métodos desarrollados para este proposito. Pollingher \& Serruya (1976). Heller (1977). Frempong (1982) y Sommer et al.. (1984) han comparado la tasa de creciniiento en dinoficeas obtenida a partir de las fases citocinéticas con la calculada mediante los procedimientos habituales. El uso de lo.;

I imnética 2: 259-264(1986)

(c) Asocia ción Lspanola de Limnología. Madrid. Spain porcentajes de células en división para estimar la verdadera tasa de creciniiento en poblaciones naturales. puede ser extensible a todo tipo de células en las que sean reconocibles estadios de dilisión.

Con respecto a las desmidiáceas. se han llevado a cabo numerosos estudios de laboratorio sobre niitosis (Brook. 1981) pero apenas se sabe lo que sucede en condiciones naturales. El presente estudio examina la disisión (sólo eri el periodo de citocinesis) de Staurastrum planctonicum var. bullosum (Desmidiaceae) en la columna vertical de un embalse cutrófico (El Vellón. Madrid) durante dos dias del mes de junio de 1983. Se pretende comparar la tasa de crecimiento calculada a partir de los cambios en la densidad de la población (donde quedarian reflejados los procesos de advección 1 sedimentación que afectan al número de células en la columna vertical), con la tasa de crecimiento obtenida a partir del tiempo de citocinesis celular. La diferencia entre ambas puede indicar cuál es 


$\begin{array}{lrrrrrr} & \mathrm{Tc}(\mathrm{h} .) & \mathrm{u}\left(\mathrm{d}^{-1}\right) & \mathrm{Td}_{1}(\mathrm{~d}) & \mathrm{r}\left(\mathrm{d}^{-1}\right) & \mathrm{Td}_{2}(\mathrm{~d}) & \mathrm{p}\left(\mathrm{d}^{-1}\right) \\ \text { (21-VI-83) } & & & & & & 0,08 \\ \text { Epilimnion } & 3,01 & 0,12 & 5,77 & 0,04 & 17,32 & -0,18 \\ 0,5 \mathrm{~m} . & 4,00 & 0,13 & 5,39 & 0,31 & 2,23 & -0,14 \\ 1 \mathrm{~m} . & 2,72 & 0,22 & 3,15 & 0,36 & 1,92 & -0,14 \\ 2 \mathrm{~m} . & 1,53 & 0,20 & 3,46 & -0,25 & 0,45 \\ 4 \mathrm{~m} . & 6,57 & 0,00 & & -1,55 & 1,55 \\ & & & & & \\ \text { (25-VI-83) } & & & & & \\ \text { Epilimnion } & 2,71 & 0,12 & 5,77 & -0,35 & 0,47 \\ 0,5 \mathrm{~m} . & 2,72 & 0,14 & 4,95 & -0,87 & 1,01 \\ 1 \mathrm{~m} . & 1,61 & 0,24 & 2,88 & -0,54 & 0,78 \\ 2 \mathrm{~m} . & 2,45 & 0,13 & 5,33 & -0,18 & 0,31 \\ 4 \mathrm{~m} . & 5,36 & 0,01 & 69,30 & -0,06 & 0,07\end{array}$

Tabla 1.-Tiempo de citocinesis (Tc). tasa de crecimiento basada cn las fases citocincticas (u). tasa de crecimiento basada cri la densidad de población $(\mathrm{r})$, tasa de pérdida $(\mathrm{p})$ y tiempos de duplicación a partir de $\mathrm{u}\left(\mathrm{Td}_{1}\right)$ y a partir de $\mathrm{r}\left(\mathrm{Td}_{2}\right)$ de Sicaurasirum planctonicum var. hullosum para el total del epilimnion del embalse y para cada una de las profundidades muestreadas.

Cytokinesic time $(\mathrm{Tc})$, cytokinesis based growth rate $(\mathrm{u})$, population based growth rate $(\mathrm{r})$, loss rate $(\mathrm{p})$, and «u» and «r» doubling times ( Td, , Td, ) of Staurastrum planctonicum var. hullosum in the entire epilimnion and at all depths.

\section{VARIABLES}

r a 1 m. ,. IA

$\mathrm{r}$ a $1 \mathrm{~m} .$, , IS

r a 4 m., , IS

r epilimnion, , IS

$\%$ c. fase A, \% c. fase C

$\%$ c. fase $\mathrm{A}, \%$ c. fase $\mathrm{B}$

$\%$ c. fase $\mathrm{B}, \%$ c. fase $\mathrm{C}$

$$
21-\mathrm{VI}-83
$$$$
\begin{aligned}
& r_{s}=0,610^{* *} \\
& r_{s}=0,700^{* *} \\
& r_{s}=0,757^{* *} \\
& r_{s}=0,622^{* *} \\
& r^{\prime}=0,737^{* *} \\
& r^{\prime}=0,648^{* *} \\
& r^{\prime}=0,795^{* *}
\end{aligned}
$$

$25-\mathrm{VI}-83$

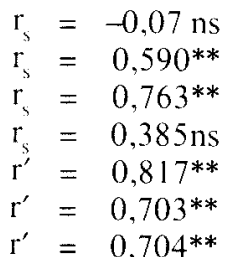

Tabla 2.-Coeficiente de correlación de Spearman $=r_{s} ; r^{\prime}=$ coeficiente de correlación de Pcarson log-log con desfasc temporal cntrc los datos igual al tiempo transcurrido entre las fases: $r=$ tasa dc crecimiento basada en la densidad de la población: IA = indicador de advección; IS = indicador de sedimentación; ${ }^{*}=$ significativopara $\mathrm{p} \leqslant 0.01 ; \mathrm{ns}=$ no significativo.

Spearman correlation coeflicient $=r_{s} ; r^{\prime}=\log -\log$ Pearson correlation coefficient with a lag in data series equal to tirnc betwcen phases; $r=$ population-based growth rate; $\mathrm{IA}=$ advection indicator; $\mathrm{IS}=$ sedimentation indicator; ${ }^{* *}=$ statistical significant at $\mathrm{p} \leqslant$ 0,$01 ; \mathrm{ns}=$ no statistical signification

la tasa de pérdida en la columna. Alvarez Cobelas (datos inéditos) observó en este mismo embalse y en la misma especie citada un ritmo diario de división cuyos máximos mitóticos tenían lugar entre las $15 \mathrm{~h}$. y las $19 \mathrm{~h}$. (hora solar).

Las características físico-químicas y biológicas del embalse de El Vellón pueden ser consultadas en Margalef et al.. (1977), Alvarez Cobelas (1982) y Alvarez Cobelas et al., (1983 y en prensa).

\section{MATERIAL Y METODOS}

Los muestreos se realizaron en el epilimnión del embalse a 0'5, 1, 2 y 4 metros de profundidad en una estación representativa de las características promedio de éste (estación B) (Alvarez Cobelas et al., 1984). Por debajo de los $4 \mathrm{~m}$., nivel en el que se situaba la termoclina no se observaron cantidades significativas de Staurastrum. Durante los días 21 y 25 de junio de 1983 se tomaron muestras mediante una bomba de succión con intervalos temporales de media hora. desde las $7 \mathrm{~h}$. hasta las $19 \mathrm{~h}$. (hora solar). Inmediatamente después de recogido el material fue fijado con formol al $4 \%$ para su conservación. Los recuentos se realizaron con un microscopio invertido siguiendo las rccomendacioncs de Rott (1981). Se contaron células en estadio vegetativo y en citocinesis. estableciéndose 
Hora solar

$21-\mathrm{VI}-83$

25-VI-83

$\begin{array}{rcccc} & \mathbf{x} & \mathbf{s} & \mathbf{x} & \mathbf{s} \\ 7 & 21,2 & 0,0 & 21,2 & 0,0 \\ 8 & 21,2 & 0,0 & 2,2,3 & 0,0 \\ 9 & 21,2 & 0,0 & 21,3 & 0,0 \\ 10 & 21,6 & 0,1 & 21,5 & 0,1 \\ 11 & 21,8 & 0,0 & 21,8 & 0,0 \\ 12 & 21,9 & 0,1 & 21,8 & 0,0 \\ 13 & 21,9 & 0,1 & 22,0 & 0,0 \\ 14 & 22,0 & 0,0 & 22,0 & 0,0 \\ 15 & 22,0 & 0,0 & 22,4 & 0,3 \\ 16 & 22,0 & 0,0 & 22,5 & 0,2 \\ 17 & 22,0 & 0,0 & 22,6 & 0,0 \\ 18 & 22,0 & 0,0 & 22,3 & 0,3 \\ 19 & 21,9 & 0,0 & 22,2 & 0,2\end{array}$

Tabla 3.-Evolución de la temperatura ("C.) durante los dos días de muestreo. $\mathrm{X}=$ valor promedio para los dos primeros metros; $\mathrm{s}=$ desviación típica.

Temperature ("C.) time course for both sampling dates: $\mathrm{x}=$ average values for the first two meters layer; $\mathrm{s}=$ standard deviation.

las siguientes categorías: (0) células en estadio vegetativo; (A) alargamiento del istmo; (B) brazos incipientes en la región del istmo: y (C) células hijas formadas pero todavía unidas.

La tasa de crecimiento basada en el porcentaje de células en citocinesis (u) se calculo mediante la fórmula de Heller (1977): $\mathrm{u}=\mathrm{Ln}([100+\mathrm{N}] / 100)$, siendo $\mathrm{N}$ el porcentaje de células que se dividen por día. obtenido a partir de la expresión: $\mathrm{N}=\mathrm{A} / \mathrm{T} c$, donde $\mathrm{A}$ es el área bajo la curva que representa el porcentaje de células en la fase A de citocinesis frente al tiempo y Tc el tiempo de citocinesis.

El tiempo de citocinesis, careciendo de máximos claros en las curvas de cada fase citocinética (figura 1), se obtuvo mediante la diferencia temporal entre los puntos de corte de las curvas de las fases A y C respectivamente, con las líneas perpendiculares a los ejes de tiempo que dividen el área bajo cada curva en dos semiáreas de igual superficie. Del mismo modo se calcularon los tiempos transcurridos entre lis fases $\mathrm{A}$ y B y entre las fases B y C.

La tasa de crecimiento basada en la densidad de la población (r), donde quedan reflejadas las variaciones debidas a procesos de advección y sedimentación, fue hallada mediante la fórmula habitual: $\mathrm{r}=(\mathrm{Ln} \mathrm{N},-\mathrm{Ln}$ $\left.\mathrm{N}_{1}\right) /\left(\mathrm{t}_{2}-\mathrm{t}_{1}\right)$; donde $\mathrm{N}, \mathrm{y} \mathrm{N}_{2}$ son las densidades de células en los tiempos $t$, y $t_{2}$ respectivamente.

Los valores de «r" que han sido comparados con «u» se hallaron para una diferencia temporal de 12 horas. mientras que los que se correlacionaron con el indicador de sedimentación y el indicador de advección se calcularon para cada hora.

Como indicadores de los procesos de advección y sedimentación se utilizaron la velocidad de la corriente a 1 metro (Hrbácek \& Straskraba, 1966) y el cociente ((densidad de células a 4 metros / densidad de células en el epilimnion», respectivamente, cuyos valores se estimaron también para cada hora.

La tasa de pérdida (p) será la diferencia entre las dos tasas de crecimiento «r» y «u» (Heller, 1977).

El tiempo de duplicación (Td) se obtuvo a partir de «u» y a partir de «r».

\section{RESULTADOS}

La tasa de crecimiento basada en las fases citocinéticas (u) para el total del epilimnion es similar durante los dos días, siendo la tasa de crecimiento basada en la densidad de la población (r) positiva y menor que «u» el día 21 y negativa el 25. Por tanto atendiendo a la tasa de pérdida (p), existe una leve pérdida el 21 y pérdida neta el 25 (tabla 1). Obsérvese sin embargo que el crecimiento real (mitosis) apenas varía de un día para otro.

El cálculo de «u» para cada una de las profundidades muestreadas (tabla 1) revela que el óptimo de crecimiento se da a 1 metro de profundidad, coincidiendo con observaciones previas (Alvarez Cobelas, datos no publicados).

La tabla 2 muestra las correlaciones existentes entre «r» (para toda la columna del epilimnion y para 1 y 4 metros de profundidad) y otras variables, como el indicador de advección y el de sedimentación. Durante el día 21 se observa ganancia de células en la columna por procesos de advección en el primer metro. 

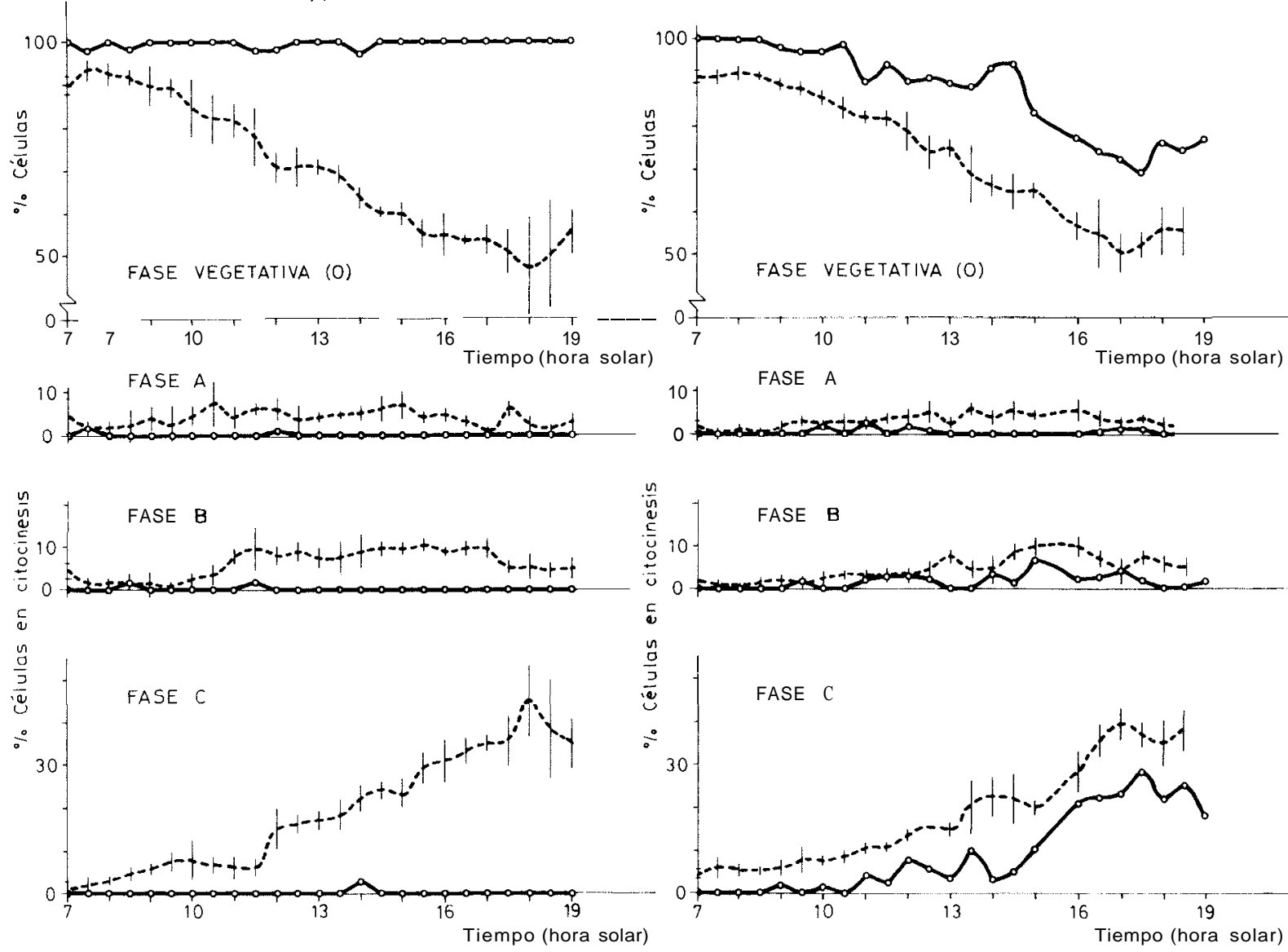

Figura 1.-Porcentaje de células en fase vegetativa (0) y eri citocinesis (fases A. B y C) durante los dias 7 l (izquierda) y 25 (derecha)de junio de 1983. Valores a $4 \mathrm{~m}$. (-). Valor promedio para $0.5,1$ y 7 ni. (--). Barras verticales: desviaciones típicas.

Percentage of cells in vegetative phase (0) and in cytokinesis (phases $A$. B y C) during 71 (left) and 25 (right) junc $1983.4 \mathrm{~m}$. values $(-)$. Average values at 0.5 .1 and 2 m. (--). Vertical hars: standard deviations.

dominando la sedimentación en la zona inferior del epilimnion. El día 25 se dieron principalmente procesos de sedimentación en toda la columna.

Existen células de Statrastrum en citocinésis durante las 24 horas del día (figura 1), pero por la tarde se produce un claro aumento en el porcentaje de células en división. alcanzando su máximo $(40 \%$ aproximadamente) entre las 17 y 18 horas.

En la tabla 1 podemos ver que el tiempo de citocinesis ( Tc) calculado para el pronicdio del epilimnión del día 21 es ligeramente superior al del día 25 , resultando en ambos casos significativa la correlación log-log entre el porcentaje de células en fase A y el porcentaje dc células en fase $C$ con un desfase temporal entre las dos series de datos igual al tiempo de cilocinesis de cada dia (tabla 2). El tiempo transcurrido entre la fase 4 y la $\mathrm{B}$ es de 77 minutos y entre las fases $\mathrm{B}$ y $\mathrm{C}$ de 103 minutos para el día 21 , mientras que para el 25 es de 100 minutos y 60 minutos respectivamente. También existen correlaciones log-log significativas para ambos dias entre los porcentajes de células de dos fases consecutivas con un desfase temporal entre los datos igual al tiempo transcurrido entre dichas fases (tabla 2).

El tiempo de duplicación a partir de «u» (tabla 1) para todo el epilimnión es similar durante los dos días de muestreo. En el cálculo por profundidades obviamente el valor mínimo se da a l metro. coincidiendo con los máximos de crecimiento. A 0,5 y 1 metro los tiempos de duplicación son superiores al dia 21 frente al 25. mientras que a 2 metros ocurre lo contrario. 


\section{DISCUSION}

La tasa de crecimiento basada en los índices mitóticos (o bien en los de citocinesis) está siendo objeto de atención en los últimos tiempos debido a que es más precisa que la calculada a partir de las densidades de las células en poblaciones naturales porque se cuantifican las células que realmente se dividen. Sin embargo, Frempong (1982) señala que la variación eri el porcentaje de células en división con la profundidad puede tener serias implicaciones en el cálculo de la tasa de crecimiento mediante este procedimiento. Tal es el caso de Staurastum planctonicum var. bullosum (cuyos máximos se dan a 1 metro), donde los resultados por profundidades son bastante diferentes entre sí. Por ello es conveniente hallar la tasa de crecimiento a partir de los porcentajes promedio de cada fase para todo el epilimnion. Si queremos conocer la tasa de crecimiento por profundidad, una fuente de error se debe a que la proporción de cada fase citocinética podría no ser similar en cada uno de los niveles por el efecto del transporte vertical (Araúzo, datos inéditos).

Debe señalarse aquí la complejidad de cualquier proceso de sedimentación en una célula con morfología tan complicada como Staurastrum (Duthie, 1965) incrementada por las variaciones en la fricción debidas al aumento de superfice de contacto con el fluido que se produce durante la citocinesis y por la probable disminución de la densidad celular en dicho proceso, observada en otras desmidiáceas (PickettHeaps, 1975). Todos estos fenómenos probablemente distorsionen en cierta medida los resultados.

La existencia de una mayor variación en la tasa de perdida que en la de crecimiento obtenida a partir de las fases citocinéticas puede estar relacionada fundamentalmente con procesos de tipo fisico, como son los efectos del viento y de los cambios de temperatura y densidad del agua, que actuan de forma variable sobre el transporte horizontal la sedimentación (Reynolds. 1984).

Respecto al tiempo de citocinésis para todo el epiliinnion. verificado mediante las correlaciones log-log entre los porcentajes de las fases inicial y final con desfase ter-tiporal igual a «TC». podemos intentar explicar su variación de un día a otro de muestreo como un efecto de las condiciones climatológicas concretas de cada dia (hipótesis sobre el efecto de la temperatura): el 21. con mayor «To» que el 25. estuvo despejado por la niañaria (bochorno) hasta las $11.30 \mathrm{~h}$. Desde esa hora hasta las $13 \mathrm{~h}$. hubo tormenta. sucediéndose periodos de tormenta alternados con claros durante la tarde. El 25 fue más estable (sin lluvia) pero no siempre despejado. En la tabla 3 vemos cómo durante el 21 el efecto de las tormentas impide que se siga calentando la parte superior del epilimnion y origina una homogenización por convección. cosa que no ocurre el 25 (Araúzo. datos inéditos). La diferencia mayor de temperatura entre los dos días se da en las horas de la tarde, cuando el número de mitosis es mayor. Como el tiempo de citocinesis es un único valor calculado para cada día no se puede buscar correlación estadística entre éste y la variación de temperatura. Sería de gran interés el cálculo de «Tc» $\mathrm{y}$ «u» en otra fase térmica del ciclo anual para comprobar si existe alguna relación entre cada uno de estos valores y la temperatura, asi como la cantidad de radiación. Esto contribuiría a esclarecer si el ritmo de división de Staurastrum es endógeno o no (Winfree, 1980).

Td, y Td, difieren notablemente, siendo mas coherentes los que fueron calculados mediante la tasa de crecimiento basada en las fases de citocinesis (Tabla 1).

No es aconsejable pues, obtener tienipos de duplicación a partir de la tasa de crecimiento basada en la densidad de células en poblaciones naturales, puesto que puede dar una idea muy distorsionada de la realidad.

Para finalizar podemos extraer también conclusiones de tipo metodológico. Los muestreos en un solo punto (columna vertical, una dimensión). no son suficientes si queremos hacer una estimación de la densidad del fitoplancton con un nivel aceptable de seguridad (véanse, por ejemplo. los resultados a los que conduce en el cálculo de «r»). Diseños experimentales en más de dos dimensiones pueden ofrecer una información más real; como puede ser el que proponen Irish \& Clarke (1984) de tipo estratificado. que aunque requiere más esfuerzo, supone también una mayor eficacia en el estudio. Por otra parte, si se pretende realizar un estudio de la dinámica del fitoplancton a escala fina es igualmente importante efectuar medidas de tipo físico (viento. radiación, densidad y viscosidad del agua, corriente. turbulencia, etc.). a la vez que recogemos datos biológicos.

\section{AGRADECIMIENTOS}

Agradecemos a Helena Guerra Criado su colaboración en el mecanografiado de este trabajo.

\section{BIBIIOGRAFIA}

Alvarez Cobelas, M. 1982. Lind limnologia de la cuenca del combalse de lil l'allon / Madrid) Tesis doetoral. Universidad Complutense. Madrid. 228 págs. + 683 págs

Alvarez Cobelas, M.; Velasco. J.L.; Rubio. A.; Colmenarejo, J.M.. 1983. Las tendencias a la cutrofiza- 
ción en el embalse de El Vellón. Actas $V$ Cong. Nul. Quimicu. Vol III. Tenerife. 707 págs.

Alvarez Cobelas, M.; Velasco, J.L.; Rubio, A.; Acosta, F.J. (en prensa). A limnology of the El Vellón reservoir in 1980 and 1981. 1. Some physico-chemical factors with special reference to horizontal patterns. Studia Oecologica.

Brook, A. J. 1981. Thr biology of desmids. Blackwell, Oxford. 280 págs.

Duthie, H.C. 1965. Some observations on the ecology of desmids. J. Ecol., 53: 695-703.

Frempong, E. 1982. The space-time resolution of phased cell division in natural populations of the freshwater dinoflagellate Ceratium hirundinella. Inr. Revue ges. Hydrobiol., 67: 323-339.

Heller, M.D. 1977. The phased division of the freshwater dinoflagellate Ceratium hirundinella and its use as a method of assessing growth in natural populations. Freshwater Biology, 7:527-533.

Hrbácek, J. \& Straskraba, M. 1966. Horizontal and vertical distribution of temperature, oxygen, $\mathrm{pH}$ and warter movements in Slapy reservoir (1958-1960). Hydrobiol, Studies, 1: 7-40.

Irish, A.E. \& Clarke, R.T. 1984. Sampling designs for the estimation of phytoplankton abundance in limnetic environments. Br. Phycol. J.. 19: 57-66.
Margalef, R.; Planas, M.D.; Armengol, J.; Vidal, A.; Prat, N.; Guisset, A.; Toja, J.; Estrada, M., 1977. Limnologia de los embalses españoles. Mopu. 422 págs. +85 págs.

Mcduff, R.E. \& Chisholm, S.W. 1982. The calculation of in situ growth rates of phytoplankton populations from fractions of cells undergoing mitosis: a clarification. Limnol. Oceanogr. 27: 783-788.

Pickett-Heaps, J.D. 1975. Green algae. Sinauer, Massachusetts. 606 págs.

Pollingher, C. \& Serruya, C. 1976. Phased division of Peridinium cinetum f. westii (Dinophyceae) and devolopment of lake kinneret (Israel) bloom. J. Phycol. 12: 163-170.

Reynolds, C.S. 1984. The ecology of freshwater phytoplankton. Cambridge University Press, Cambridge. 384 págs.

Rott, E. 1981. Some results from phytoplankton couniing intercalibrations. Schweiz. Z. Hydrol., 43: 34-62.

Sommer, U.; Wedemeyer, C.; Lowsky, B.. 1984. Comparation of potential growth rates of Ceratium hirundinella with observed population density changes. Hydrobiologia, 109: 159-164.

Winfree, A.T. 1980. The geometry of biological time. Spring Verlang, Berlin. 530 pág. 